\title{
Osteocalcin Hormone is a New Important Concern for the Aging Population
}

\author{
Ying Gao Balch* \\ Department of Human Sciences, The University of Arkansas at Pine Bluff, USA
}

*Corresponding author: Ying Gao Balch, Department of Human Sciences, The University of Arkansas at Pine Bluff, USA.

\begin{abstract}
A new study has found out that our human skeletal system is also an endocrine organ which induces the hormone called osteocalcin and then transforms it into the body's blood. Hormone-osteocalcin interacts with other tissue and organ hormones to reach an optimal goal for daily activity. The question is, how we can make more osteocalcin, there are two ways- physical activity and proper nutrition.
\end{abstract}

Keywords: Osteocalcin; Physical Activity; Nutrition

\section{Introduction}

The human bone was thought to be just a simple organ that is a mineralized tissue to protect the body's organs. Today's studies have demonstrated that bone acts as an endocrine organ, and then involved in interaction to change communication with other tissues in the body that was not understood before [1]. The body tissue or organs each other interact through the blood of the hormones. We know the bone is very much a live organ, and bone is one of the participating network signals to other organs by bone to produce their own Hormone-osteocalcin circulating in the body's blood [2]. Hormone-osteocalcin plays a crucial role in bone remodeling that enables our bone to grow and recover from injuries [1]. Osteocalcin is signaling in the brainstem, thalamus, and hypothalamus through the blood-brain barrier (BBB). Those areas are influencing various neurotransmitters synthesis and signaling to increase serotonin synthesis [3]. New discoveries have proved the bone mass reduction may also be linked to the weakening of muscles and memory and cognitive issues [3].

Ongoing research in this area suggests staying physically active regularly, and then it stimulates your bone to make more osteocalcin, which will have these beneficial effects on muscle and brain [4]. Osteocalcin naturally declines at the beginning of women at age 30 and men at age 50 [5]. We should reinforce the message to the people to be active during adolescence and early adult years, and this means they reach a higher peak bone mass, which will protect them from age-related problems linked to osteocalcin decline [6]. These findings can support public health messages regarding the importance of staying active through middle age and later life. Epidemiological studies have shown that very active people tend to have less cognitive decline with aging than a sedentary lifestyle. Maintaining physical activity has long been known to have antiaging properties. With time, we understand its related functions could increase more awareness in public health intervention in the future.

Restoring osteocalcin levels reverses age-related decline in exercise capability. However, be aware of daily eating patterns to increase serum osteocalcin levels. When we closely look at food resources related to increased osteocalcin of nutrients in your blood. The information is about healthy bone-supporting nutrients that will play an important role in bone health [7]. The nutrients can be found in various foods, especially dark, leafy green vegetables: mustard greens, swiss chard, collard greens, spinach, broccoli, brussels sprouts, green beans, prunes, kiwi fruit, soybean oil, avocado, and green peas. We can also find animal food resources, such as hard and soft cheese, egg yolk, ground beef, beef liver, pork chops, chicken, goose, and fermented plant foods, such as Asian 
natto. All of the facts have proven to increased serum osteocalcin levels. That is, focuses on balance to establish a maximum efficacy nutrient to give your body extra protection and healthy lifestyle behaviors can help you and your family stay a step ahead.

\section{References}

1. Shen Y, Xu Y, Bao Y (2020) Interaction among skeleton, body fat and cardiovascular diseases mediated by osteocalcin. Obesity Medicine 17.

2. Mizokami A, Kawakubo Yasukochi T, Hirata M (2017) Osteocalcin and its endocrine functions. Biochemical Pharmacology 132:1-8.

3. Zoch M, Clemens T, Riddle R (2016) New insights into the biology of osteocalcin. Bone 82: 42-49.
4. Mera P, Laue K, Ferron M, Confavreux C, Wei J, et al. (2017) Erratum: Osteocalcin Signaling in Myofibers Is Necessary and Sufficient for Optimum Adaptation to Exercise. Cell metabolism 25(1): 218.

5. Smith C, Voisin S, Al Saedi A, Phu S, Brennan Speranza T (2020) Osteocalcin and its forms across the lifespan in adult men. Bone 130: 115085 .

6. Chahla S, Frohnert B, Thomas W, Kelly A, Nathan B, et al. (2015). Higher daily physical activity is associated with higher osteocalcin levels in adolescents. Preventive Medicine Reports 2: 568-571.

7. Blondell SJ, Hammersley Mather R, Veerman JL (2014) Does physical activity prevent cognitive decline and dementia? A systematic review and meta-analysis of longitudinal studies. BMC Public Health 14: 510.

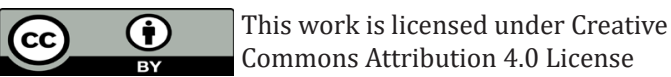

To Submit Your Article Click Here:

Submit Article

DOI: $10.32474 /$ RRHOAJ.2020.05.000218

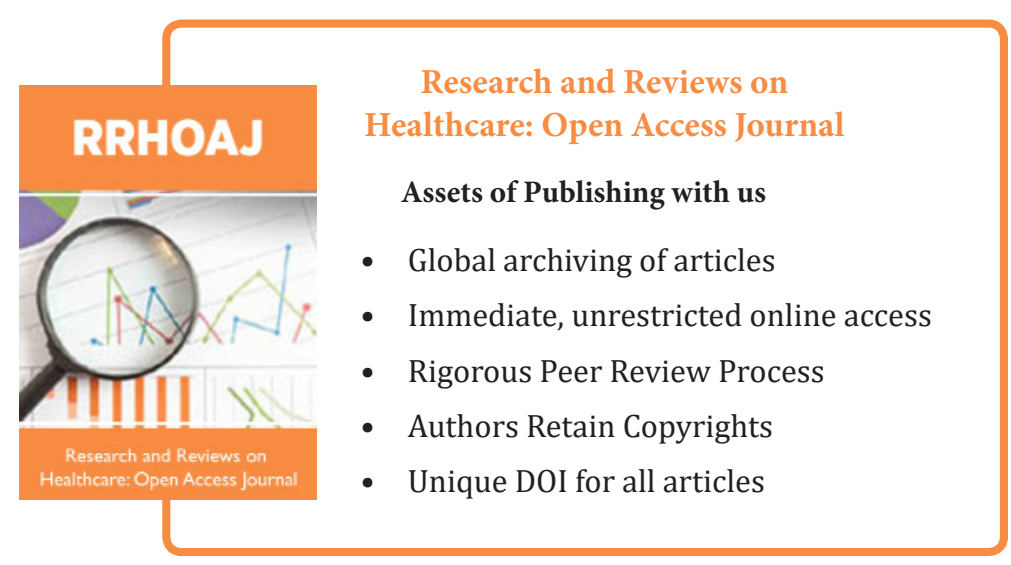

